\title{
Direct Evidence for Projectile Charge-State Dependent Crater Formation Due to Fast Ions
}

\author{
R. M. Papaléo, ${ }^{1}$ M. R. Silva, ${ }^{1}$ R. Leal, ${ }^{1,2}$ P. L. Grande, ${ }^{2}$ M. Roth, ${ }^{3}$ B. Schattat, ${ }^{3}$ and G. Schiwietz ${ }^{3}$ \\ ${ }^{1}$ Faculty of Physics, Catholic University of Rio Grande do Sul, Av. Ipiranga 6681, C.P. 1429, 90619-900 Porto Alegre, Brazil \\ ${ }^{2}$ Instituto de Física da Universidade Federal do Rio Grande do Sul, \\ Avenida Bento Gonçalves 9500, 91501 - 970, Porto Alegre, RS, Brazil \\ ${ }^{3}$ Hahn-Meitner Institut, Bereich SF, Glienicker Strasse 100, 14109 Berlin, Germany
}

(Received 3 March 2008; published 16 October 2008)

\begin{abstract}
We report on craters formed by individual $3 \mathrm{MeV} / u \mathrm{Au}^{q_{\text {ini }}+}$ ions of selected incident charge states $q_{\text {ini }}$ penetrating thin layers of poly(methyl methacrylate). Holes and raised regions are formed around the region of the impact, with sizes that depend strongly and differently on $q_{\text {ini }}$. Variation of $q_{\text {ini }}$, of the film thickness and of the angle of incidence allows us to extract information about the depth of origin contributing to different crater features.
\end{abstract}

DOI: 10.1103/PhysRevLett.101.167601

Energetic atomic [1-3], molecular or cluster ions [4] impacting solids may leave tiny holes at the surface often surrounded by a raised region of displaced material. The observed surface morphology [1-4] is similar to what is found in ablation craters produced by an intense laser pulse $[5,6]$, in macroscopic craters produced by meteorite impacts on planets [7], or by balls dropped into granular media [8], although their spatial scales may differ by about 17 orders of magnitude. Depending on the energy regime of the ions and the type of material being bombarded, the shape of the impact features and the underlying mechanisms of formation may differ $[9,10]$. As the energy deposited by swift ions of equal kinetic energy, but different charge-states may vary substantially close to the surface, a detailed knowledge of charge-state dependent effects is of great importance for ion-beam based techniques of materials structuring (such as ion-track etching), particularly considering the new demands for smaller pattern sizes and the use of thinner layers [11]. Our results give direct evidence for a strong dependence of the surface modifications introduced by single fast ions on their charge state. For track-etching procedures, this means it is possible to control the etching sensitivity at the surface and charge equilibration below the surface should yield different etched shapes, dependent on the incident charge state. Moreover, by employing a series of well-defined nonequilibrium charge states, we could derive depth information on the near-surface effects induced by single fast ion impacts.

In this Letter, we focus on cratering induced by high velocity ions. At specific kinetic energies of a few $\mathrm{MeV}$ per nucleon, such ions transfer more than $99 \%$ of their energy to the target-electron system. A considerable fraction of the energy deposited in the track of a swift heavy ion is concentrated in a core region ( $\approx 1 \mathrm{~nm}$ ), where ionizations are directly produced by the ions. Fast secondary electrons spread the rest of the energy over larger distances. The exact size of such regions depend on the material and on the velocity of the ions, while the total amount of energy
PACS numbers: 79.20.Rf, 61.80.Jh, 61.82.Pv, 81.16.Rf

loss per path length, $S_{e}=d E_{e} / d x$, is well understood [12]. The subsequent electron dynamics, however, is a nonlinear phenomenon and it may result in large sputter yields and cratering, due to the coupling of electronic and atomic degrees of freedom [13-15]. For reviews on ion-induced electronic cratering processes or surface tracks the reader is referred to $[16,17]$.

The common way to vary the deposited energy inside the ion tracks is to change the speed or the atomic number $Z$ of the ions. This was employed in several cratering studies $[17,18]$. Using projectiles with the same $Z$ and velocity but with selected initial charge states $q_{\text {ini }}$ is, however, a cleaner method to investigate the role of the deposited energy density close to the surface, since the excitation volume and the type of ion are fixed and the deposited energy can be tuned by the selection of the initial charge [12]. Indeed, the ion charge state changes from its initial value $q_{\text {ini }}$ to a mean value $q_{\text {mean }}(l)$ as a function of the path length $l$ inside the solid. The charge-state equilibrium for such fast ions is determined by the balance between electron capture and electron loss rates, during penetration in the bulk (decay processes are not so important) [19]. For thin films, the condition $q_{\text {mean }}(l) \approx q_{\text {ini }}$ can be achieved throughout the entire layer. Thus, we have used thin layers of a polymer and $593 \mathrm{MeV} \mathrm{Au}$ ions to meet this condition. As a reference, we have also used thicker foils and tilted incidence, for which the effects are dominated by the equilibrium charge-state $q_{\text {eq }}$.

The heavy-ion cyclotron at the Hahn-Meitner Institute was used to produce a $3 \mathrm{MeV} / u^{197} \mathrm{Au}^{30+}(593 \mathrm{MeV}$ ) ion beam. Using optimized post-cyclotron carbon-stripper foils, different charge states were produced and selected magnetically. The experimental mean equilibrium charge state for this case is $q_{\mathrm{eq}}=46.3 \pm 0.1$ and the equivalent half equilibration-depth in poly(methyl methacrylate) (PMMA) is $l_{\mathrm{eq}} \approx 100 \mathrm{~nm}$ (300 nm for $90 \%$ equilibration). $S_{e}$ was calculated for each investigated charge state, using the CASP 3.2 program [20], resulting approximately in $S_{e} \propto q_{\text {ini }}^{1.67}$. 

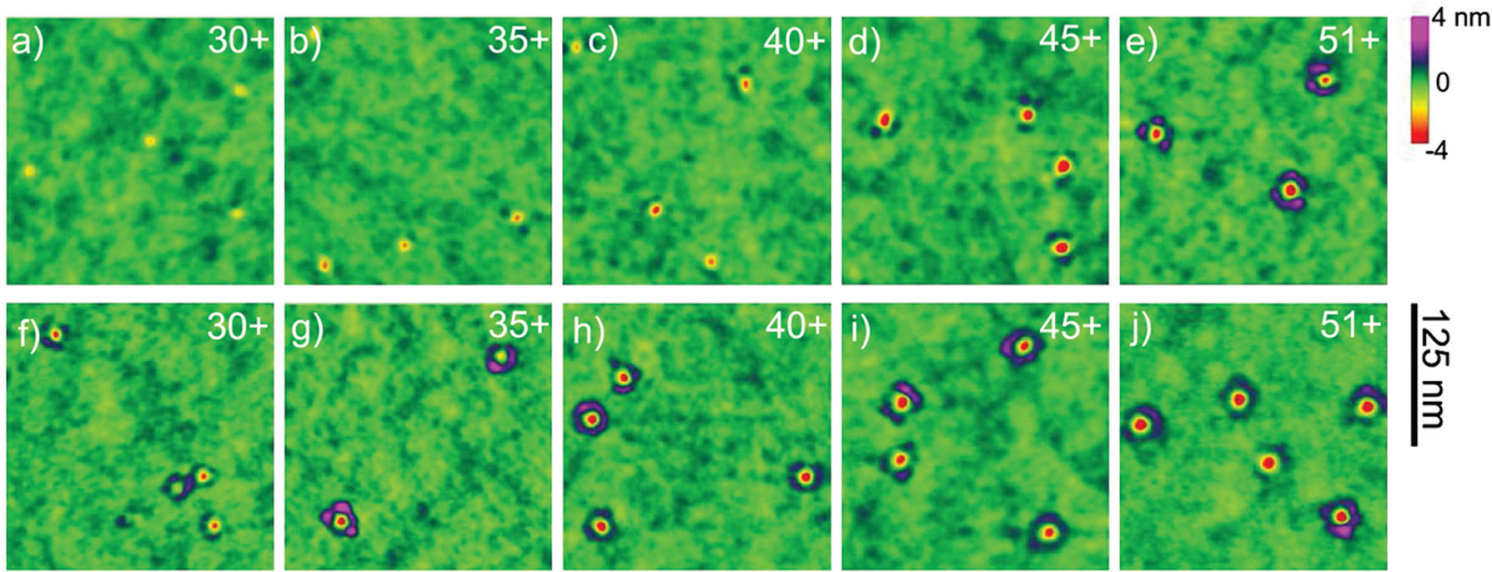

FIG. 1 (color). SFM images of PMMA thin films of two thicknesses, bombarded at normal incidence by $3 \mathrm{MeV} / u$ Au ${ }^{q_{\text {ini }}}{ }^{+}$of different initial charge states: $(\mathrm{a}, \mathrm{f}) 30+,(\mathrm{b}, \mathrm{g}) 35+,(\mathrm{c}, \mathrm{h}) 40+,(\mathrm{d}, \mathrm{i}) 45+,(\mathrm{e}, \mathrm{j}) 51+$. Images (a) to (e) are for $10 \mathrm{~nm}$ thick films, while (f) to (j) are for $105 \mathrm{~nm}$ thick layers. See Ref. [21] for a zoomed view.

Thin films of PMMA spin-coated from anisol solutions in two different thickness ranges (9-11 $\mathrm{nm}$ and 100 $110 \mathrm{~nm}$ ) onto a $\mathrm{Si}$ substrate were bombarded with $3 \mathrm{MeV} / u \mathrm{Au}^{q_{\text {ini }}+}$ beams $\left(q_{\text {ini }}=30,35,40,45\right.$, and 51). Two distinct angles of incidence $\left(0^{\circ}\right.$ and $79^{\circ}$ to the surface normal) were employed. The size and shape of the surface tracks were characterized offline with a Nanoscope IIIa (Digital) scanning force microscope (SFM) in the intermittent contact mode at a scan frequency of $1-2 \mathrm{~Hz}$ and 256-512 scan lines per image. Only images collected with high quality Si tips were stored and used for quantitative evaluation of the data. Special care was taken of using the same tip (or at least tips of similar quality) to image a set of samples covering the complete range of charge states (for details see [16]).

Typical images of impact features at normal and grazing incidence are shown in Figs. 1 and 2, respectively. The bombardments of the $10 \mathrm{~nm}$ layers at normal incidence are most appropriate to evaluate the dependence of the impact features on $q_{\text {ini }}$, because the charge state does not vary significantly inside a film of thickness $t$ with $t \ll l_{\text {eq }}$. Figures 1(a)-1(e) show a series of SFM images of PMMA films bombarded under such conditions. Craters are observed even at the lowest charge state on the $10 \mathrm{~nm}$ layers with $100 \%$ efficiency (one hole per incident ion). Ridges (raised regions), clearly distinguishable from the natural roughness of the films, are only observed at the highest charge states. In contrast, for the thick films [Figs. 1(f)-1(j)] ridges are seen with high efficiency for all $q$. Figure 2 shows in addition elongated craters due to the inclined incidence and pronounced tail structures extending along the direction of ion penetration.

Figure 3 depicts quantitative data on the averaged sizes of crater depressions (holes) and ridges (all elevated surfaces, consisting of raised rims plus tails) as a function of $q_{\text {ini }}$, thickness $t$ and angle of incidence $\theta_{\text {ini. }}$. Each data point is a mean value over about 100 impact features. The bars in
Fig. 3 (symmetric on a linear scale) are not error bars, but reflect the size fluctuations for individual impacts, which are often large $(\sim 30 \%)$, because of the statistical nature of the energy dissipation via electron-collision cascades. Such fluctuations are not the result of using tips of distinct sharpness; they are present in every SFM image. The net volume of the raised regions in Fig. 3(a) was determined by the difference of the positive ridge volume fraction and a corresponding roughness volume, both related to a reference plane positioned at the rms height of the unperturbed surface $(\approx 0.4 \mathrm{~nm})$. The mean roughness volume across

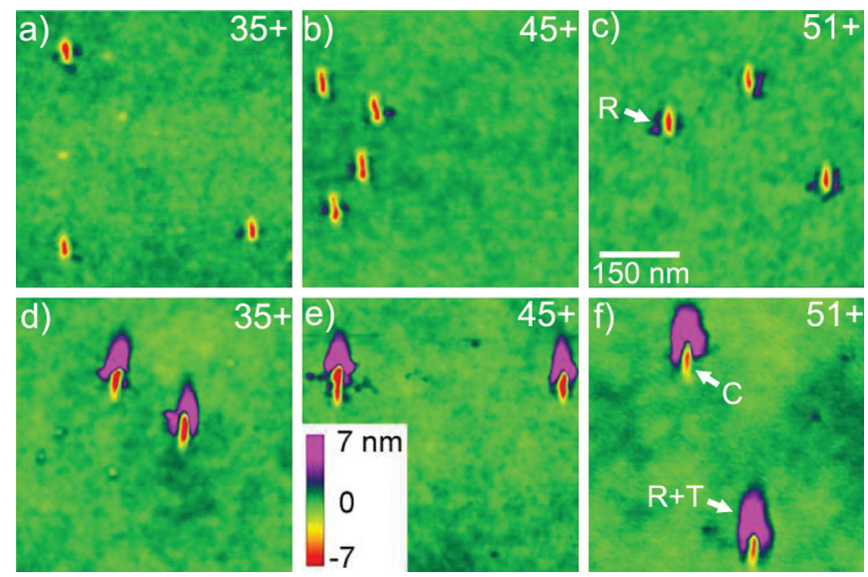

FIG. 2 (color). SFM images of PMMA thin films bombarded at grazing angles $\left(\theta_{\text {ini }}=79^{\circ}\right)$ with $3 \mathrm{MeV} / u \mathrm{Au}^{q_{\text {ini }}+}$ of different charge states. Images (a) to (c) are obtained for $10 \mathrm{~nm}$ thick films, and images (d) to (f) for $105 \mathrm{~nm}$ layers. The capital letters indicate different crater constituents. $C$ stands for the central crater hole, $R$ is the surrounding $\operatorname{rim}$ and $T$ is the asymmetric tail (for tilted incidence, all structures are elongated). The ridge volume is the sum of all protrusions (positive volume fractions $R+T$ ). Contrary to the rims, the tails are absent on the thin layers, indicating the importance of deep layers above the $\mathrm{Si}$ substrate. 


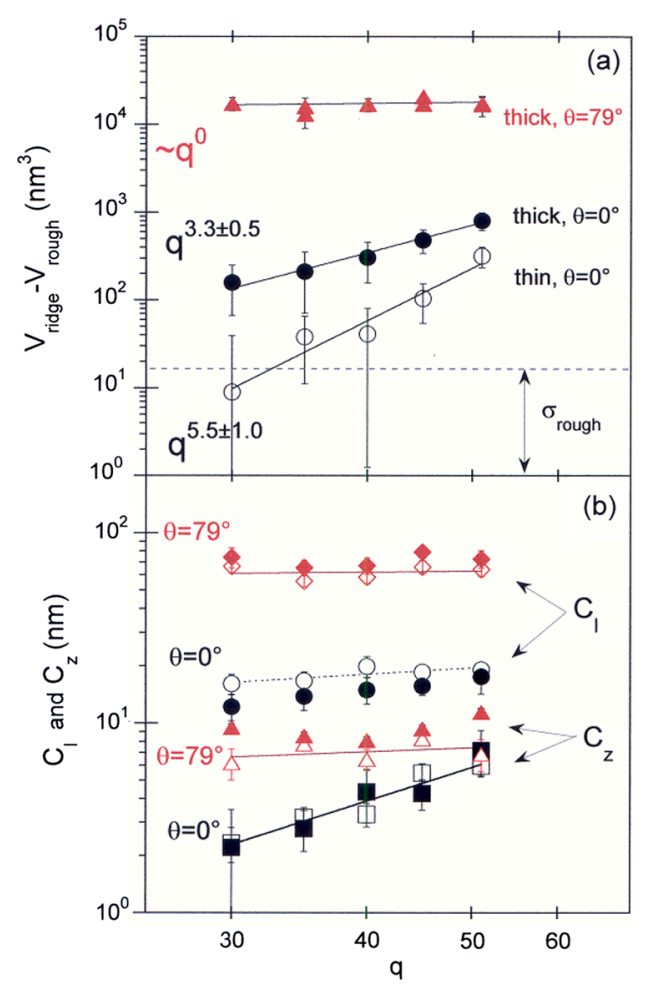

FIG. 3 (color online). Averaged crater dimensions on PMMA as a function of entrance charge state for normal and for grazing angles of incidence. Data for the thin layers (10 nm-thick films) are shown as open symbols and for the thick films $(105 \mathrm{~nm})$ as closed symbols. (a) Average ridge volume (rim plus tail). As no tails are seen at grazing angles on the thin films, just the data for the $105 \mathrm{~nm}$ films are shown. (b) Average length $C_{l}$ and depth $C_{z}$ of the depressed crater surfaces. The crater depth is measured relative to the unperturbed surface height. All solid lines are power fits to the experimental data.

this reference plane $V_{\text {rough }}$ is $\approx 16 \mathrm{~nm}^{3}$, with a dispersion $\sigma_{\text {rough }} \approx 16 \mathrm{~nm}^{3}$, as indicated by the dashed line in Fig. 3 (a). At normal incidence, the net volume of the raised regions for low charge states is very close to the volume due to pure roughness. In the following, we discuss the normal-incidence results first, before we consider the tilted geometry.

As can be seen from Fig. 3(a), for the thin layers we find a strong increase of the ridge volume with increasing $q_{\text {ini }}$. In particular, $V_{\text {ridge }} \propto q_{\text {ini }}^{5.5 \pm 1.0}$, what gives roughly a cubic dependence of the ridge volume on the deposited energy density in the central track. For the $105 \mathrm{~nm}$ layers, the charge state changes towards equilibrium inside the film and the dependence on $q_{\text {ini }}$ is expected to be smeared out. In fact, a weaker ( $\propto q_{\text {ini }}^{3}$ ) dependence of the ridge volume is observed for the thick films. Because of the finite size of the SFM tip, which affects mainly the smaller protrusions at low $q$ and may prevent complete access to the deeper holes at high $q$, all plotted scalings may be slightly steeper, but certainly not weaker than the values given above.

In addition, we can estimate the depth of the excited region contributing to the ridge formation (the depth of origin) from the ratio of the ridge volumes for the two films' thicknesses at normal incidence in Fig. 3(a), considering only the selected (equilibrium) projectile charge $q_{\text {ini }}=45$ (no charge-equilibration effect). This ratio $(\approx 6)$ leads to a depth of origin of about $50 \mathrm{~nm}$, as has been estimated assuming an exponential attenuation of the excitation energy with depth. This relatively large value is also supported by the weaker charge-state dependence found for ridges on the thicker films.

The behavior of the crater depressions is distinctively different from the ridges. No major differences were found between the thin and the thick layers, both for the hole depth and for the length. The hole diameter $\left(C_{l}\right)$ and thus the crater area shows only a very weak variation with $q_{\text {ini }}$, whereas the depth behaves as $C_{z} \propto q^{1.8 \pm 0.3}$. Thus, the charge-state variation of the hole volume is much weaker $\left(\propto q_{\text {ini }}^{3}\right)$ than for the ridge volume. The absence of a thickness effect (within the error bars) for the hole dimensions $\left(C_{l}\right.$ and $\left.C_{z}\right)$ shows that hole formation is dominated by near-surface events, occurring at distances smaller than $10 \mathrm{~nm}$ below the surface.

At a grazing incidence, we observe nearly no influence of $q_{\text {ini }}$ on cratering [see Fig. 3(b)]. In principle, a close surface proximity of regions excited under charge-state equilibrium conditions could be responsible for this effect. Nevertheless, at distances smaller than $10 \mathrm{~nm}$ below the surface (where the hole formation should occur), the ion path length would be smaller than $50 \mathrm{~nm}$, which is below the charge-equilibration distance $\left(l_{\text {eq }} \approx 100 \mathrm{~nm}\right)$. Thus, the charge-equilibration process cannot totally explain the weak $q_{\text {ini }}$ dependence found for the grazing incidence.

Theoretically, it is clear that track effects are triggered by electronic excitation and ionization, which relax rapidly into atomic motion. Intense pressure, sound or shock waves [22] may lead to a collective and correlated motion and possible rearrangement of all atoms close to the track. In addition, thermal-spike effects invoked by nonequilibrium potentials or by hot electrons [23,24] or the late phase of the Coulomb-explosion spike [23,25] (a mutual repulsion of target ions) may lead to a disordered atomic motion. Depending on the electronic heat and electron density, the plasma dynamics [6] or a gas flow out of the ion track at high atomic temperatures (as discussed also for macroscopic volcano eruptions [26]) may also be important. One may speculate that the inner part of the crater is shaped by short-ranged effects such as melting and evaporation in the thermal spike, whereas the ridge might be related to interactions of longer range such as a pressure pulse [22]. However, an influence of long range stress fields after rapid cooling of molten tracks (the so-called ion-hammering effect) cannot be excluded, as shown qualitatively for hillock formation in vitreous silica [24].

In the short-time models for atom dynamics, it is mainly the energy density close to the surface, which controls particle ejection and cratering. On purpose, however, we avoid using our experimental charge-state scalings as a test 
of the reliability of specific models, because different versions of the same type of model predict different scalings and different types of models (e.g. thermal or hydrodynamical) may predict the same scaling [22,27,28]. A quantitative modeling of the complex process of crater formation by fast atomic projectiles is far from being accomplished. Molecular dynamic simulations [29] are the most promising approach to check an adopted model for the electronic energy-transfer channel, as they include all atomic degrees of freedom. Thus, they show, e.g., the importance of atomic heat transport and of melting in the track, and of pressure waves extending further outside [29]; even though, quantitative results are far from being satisfactory. We finally note similar charge-state effects have been recently observed for slow highly-charged ions [9] on inorganic crystals (only raised regions were seen in this case). For slow ions, energy is transferred mainly via the potential energy of the ions; for fast ions, kinetic transfer dominates. This might be an indication that the subsequent processes of energy transport and dissipation after a single ion impact, which eventually lead to the topological changes, have little memory of the way energy is initially delivered at the surface.

In summary, we found direct evidence for a strong dependence of the surface modifications introduced by single fast ions on the projectile charge state. Craters and ridges formed in PMMA, though clearly interwoven structures, depend very differently on $q_{\text {ini }}$ (and hence on the energy deposited near the surface). Comparison of the different experimental data sets indicates also that the excitation depth contributing to the ridge formation $(\sim 50 \mathrm{~nm})$ is much larger than the one involved in the hole formation $(<10 \mathrm{~nm}$ below the surface). Our findings imply that patterning produced directly by single fast ions and pore shaping by a subsequent etching step may be improved, by properly tuning the initial charge state of the projectiles. It is also proven that the use of tabulated equilibrium energy losses, the common practice in the field, is not adequate for describing surface modifications. The present work calls for further detailed measurements of the topological changes induced by single ion impacts on surfaces as a function of the layer thickness and type of substrate, on a way to a more profound understanding of ion-track processes at surfaces.

The Brazilian agencies CAPES and CNPq, and DAAD (Germany) are acknowledged for financial support.

[1] R. M. Feenstra and G. S. Oehrlein, Appl. Phys. Lett. 47, 97 (1985).

[2] I. H. Wilson, Surf. Interface Anal. 20, 637 (1993).

[3] R. C. Birtcher, S. E. Donnelly, and S. Schlutig, Phys. Rev. Lett. 85, 4968 (2000).

[4] Z. Insepov, R. Manory, J. Matsuo, and I. Yamada, Phys. Rev. B 61, 8744 (2000).
[5] R. Stoian, M. Boyle, A. Thoss, A. Rosenfeld, G. Korn, I. V. Hertel, and E. E. B. Campbell, Appl. Phys. Lett. 80, 353 (2002).

[6] Lan Jiang and Hai-Lung Tsai, Appl. Phys. Lett. 87, 151104 (2005).

[7] M. C. Malin, K. S. Edgett, L. V. Posiolova, S. M. McColley, and E. Z. Noe Dobrea, Science 314, 1573 (2006).

[8] A. M. Walsh, K. E. Holloway, P. Habdas, and J. R. de Bruyn, Phys. Rev. Lett. 91, 104301 (2003).

[9] A. S. El-Said, R. Heller, W. Meissl, R. Ritter, S. Facsko, C. Lemell, B. Solleder, I. C. Gebeshuber, G. Betz, M. Toulemonde, W. Möller, J. Burgdörfer, and F. Aumayr, Phys. Rev. Lett. 100, 237601 (2008).

[10] J. Samela and K. Nordlund, Phys. Rev. Lett. 101, 027601 (2008).

[11] C. Harrell, Z. Siwy, and C. Martin, Small 2, 194 (2006).

[12] P. L. Grande and G. Schiwietz, Nucl. Instrum. Methods Phys. Res., Sect. B 195, 55 (2002); in Advances in Quantum Chemistry, edited by J. Sabin (Elsevier, New York, 2004), Vol. 45, pp. 7-46.

[13] F. Thibaudau, J. Cousty, E. Balanzat, and S. Bouffard, Phys. Rev. Lett. 67, 1582 (1991).

[14] J. Kopniczky, C. T. Reimann, A. Hallén, B. U. R. Sundqvist, P. Tengvall, and R. Erlandsson, Phys. Rev. B 49, 625 (1994).

[15] L. S. Farenzena, R. P. Livi, M. A. de Araújo, G. G. Bermudez, and R. M. Papaléo, Phys. Rev. B 63, 104108 (2001).

[16] Ion Irradiation of Polymers: Fundamentals and Applications, edited by D. Fink (Springer, Berlin, 2004), Vol. 1.

[17] R. Neumann, Nucl. Instrum. Methods Phys. Res., Sect. B 151, 42 (1999).

[18] R. M. Papaléo, L. S. Farenzena, M. A. de. Araújo, R. P. Livi, M. Alurralde, and G. Bermudez, Nucl. Instrum. Methods Phys. Res., Sect. B 148, 126 (1999).

[19] H.-D. Betz, Rev. Mod. Phys. 44, 465 (1972).

[20] The CASP 3.2 program is available at http://www.hmi.de/ people/schiwietz/casp.html.

[21] See EPAPS Document No. E-PRLTAO-101-065842 for a zoomed view of SFM images presented in Figs. 1(a)-1(e) of the manuscript. For more information on EPAPS, see http://www.aip.org/pubservs/epaps.html.

[22] D. Fenyo and R. E. Johnson, Phys. Rev. B 46, 5090 (1992); R. E. Johnson, B. U.R. Sundqvist, A. Hedin, and D. Fenyo, Phys. Rev. B 40, 49 (1989).

[23] G. Schiwietz, K. Czerski, M. Roth, F. Staufenbiel, and P. L. Grande, Nucl. Instrum. Methods Phys. Res., Sect. B 225, 4 (2004); 226, 683 (2004); Phys. Rev. Lett. 99, 197602 (2007).

[24] S. Klaumünzer, K. Dan. Vidensk. Selsk. Mat. Fys. Medd 52, 293 (2006).

[25] R. L. Fleischer, P. B. Price, and R. M. Walker, Nuclear Tracks in Solids (Universiy of California Press, Berkeley, 1975).

[26] A. B. Clarke, B. Voight, A. Neri, and G. Macedonio, Nature (London) 415, 897 (2002).

[27] M. Urbassek and P. Sigmund, Appl. Phys. A 35, 19 (1984).

[28] I. S. Bitensky and E. S. Parilis, Nucl. Instrum. Methods Phys. Res., Sect. B 21, 26 (1987).

[29] E. M. Bringa, R.E. Johnson, and R. M. Papaléo, Phys. Rev. B 65, 094113 (2002); E. M. Bringa (private communication). 